\title{
CCN1 expression in interleukin-6 deficient mouse kidney in experimental model of heart failure
}

\author{
Tomasz Andrzej Bonda ${ }^{1}$, Andrzej Taranta ${ }^{1}$, Karol Adam Kaminski ${ }^{2}$, \\ Magdalena Dziemidowicz $^{1}$, Sergey Litvinovich ${ }^{5}$, Marcin Kozuch ${ }^{3}$, Izabela Bialuk ${ }^{1}$, \\ Lech Chyczewski ${ }^{4}$, Maria Malgorzata Winnicka ${ }^{1}$
}

\author{
${ }^{1}$ Department of General and Experimental Pathology, Medical University of Bialystok \\ ${ }^{2}$ Department of Cardiology, Medical University of Bialystok \\ ${ }^{3}$ Department of Invasive Cardiology, Medical University of Bialystok \\ ${ }^{4}$ Department of Medical Pathomorphology, Medical University of Bialystok \\ ${ }^{5}$ Department of Hospital Therapy, Grodno State Medical University, Belarus
}

\begin{abstract}
Chronic heart failure often leads to worsening of the renal function. Mediators of this process include inflammatory and neuroendocrine factors. CCN1 (Cyr 61), a member of growth factor-inducible immediate early genes, which modulates inflammation and fibrogenesis, is excreted with urine in the early phase of acute renal injury and may be involved in the pathogenesis of the cardiorenal syndrome. The aim of the study was to evaluate CCN1 protein abundance and localization in the kidney of IL-6-deficient C57BL/6J (IL-6 KO) mice and respective wild-type (WT) animals in basal conditions and in animals with chronic heart failure twelve weeks after myocardial infarction. Age- and sex-matched mice from both strains subjected to sham operation served as controls. One group of WT animals subjected to myocardial infarction was treated with antagonist of AT1 receptor telmisartan over 12 weeks. Abundance and localization of CCN1 protein in kidney were assessed with Western blotting and immunohistochemistry, respectively. In all groups the strongest immunohistochemical reaction for CCN1 was observed in distal convoluted tubules and in smaller arteries, however, the total expression of CCN1 protein was lower in IL-6 KO mice in comparison to WT animals. The main difference in CCN1 distribution between the examined genotypes was lack of reaction in internal renal medulla and very weak reaction in proximal convoluted tubules in IL-6 KO mice. Experimental heart failure only slightly attenuated the expression of CCN1 protein in the kidney of WT mice and had no effect in IL-6 KO mice. Although, blockade of AT1 receptor did not alter CCN1 protein expression in kidneys of WT mice after myocardial infarction, it significantly changed its CCN1 distribution in the renal tubular system. (Folia Histochemica et Cytobiologica 2013, Vol. 51, No. 1, 84-91)
\end{abstract}

Key words: CCN1, kidney, heart failure, IL-6 deficient mice, telmisartan

\section{Introduction}

The kidney controls the volume of blood that is required for proper perfusion of organs. Primary dys-

\footnotetext{
Correspondence address: M.M. Winnicka

Department of General and Experimental Pathology,

Medical University of Białystok

A. Mickiewicza St. 2C; 15-222 Bialystok; Poland;

tel.: +48 857485 593;

e-mail: patofiz@umb.edu.pl
}

function of either the kidney or the heart may initiate impairment of the other, which has been described as the cardiorenal syndrome [1]. Acute worsening of cardiac performance leading to deterioration of renal function is classified as type 1 , and chronic heart failure causing progressive kidney dysfunction as type 2 of the cardiorenal syndrome [1]. Interactions between the heart and the kidney seem to be multidirectional and involve hemodynamic, neurohormonal and inflammatory factors. Myocardial infarction was shown to worsen kidney function in clinical as well as 
in experimental conditions [2, 3]. Glomerular filtration rate declines early following myocardial infarction and persists as a chronic abnormality and is accompanied by initial transient macrophage infiltration and progressive fibrosis of the renal interstitium. Upregulation of proinflammatory interleukin 6 (IL-6) after myocardial infarction [3, 4], in addition to the induction of other proinflammatory cytokines, compromises cardiac performance, and is postulated to be one of early predictive factors for secondary acute kidney injury [5]. In chronic heart failure (CHF), level of IL-6 is elevated proportionally to the severity of circulatory insufficiency [6], and is an independent predictor of mortality [7]. Increased plasma IL-6 concentration was also described in chronic kidney disease [8]. After acute myocardial infarction the level of IL-6 in plasma of patients with previous renal dysfunction is more increased than in those with well preserved kidney function [9]. This underlies the importance of this cytokine and inflammatory mechanisms in general in the pathogenesis of chronic cardiorenal syndrome. IL-6 evokes cellular responses via its membrane receptor and the two main downstream pathways: JAK2/STAT3 and MAPK/ERK [10] and activation of JAK2/STAT3 was previously reported to induce CCN1 expression [11]. CCN1 (also known as Cyr 61 - cysteine rich protein 61) belongs to the $\mathrm{CCN}$ family (the acronym derived from first letters of the three members first discovered: Cyr61, CTGF (connective tissue growth factor), and NOV (nephroblastoma overexpressed) that comprises six secreted proteins grouped together on the basis of a similar modular secondary structure [12]. CCN1 is a multifunctional matricellular protein, that plays essential roles in cardiovascular development, wound healing, fibrogenesis and regulation of inflammatory process [13]. Muramatsu et al. showed that experimental renal ischemia in rats and mice induces upregulation of $\mathrm{CCN} 1$, mainly in the proximal straight tubules, and its elevated urine level was postulated to be a marker of renal ischemia/reperfusion injury [14]. The present knowledge about the regulation and the role of $\mathrm{CCN} 1$ in healthy and diseased kidneys is very scant. In rodent kidney the basal expression of CCN1 protein is rather low [14]. In rat kidney it was observed in the outer stripe of outer medulla and in some afferent and efferent arterioles, while experimental anti-Thy1 glomerulonephritis induced $\mathrm{CCN} 1$ expression in podocytes [15]. In different tissues the expression of $\mathrm{CCN} 1$ can be regulated by over 30 factors [16]. In the heart and in the vasculature, angiotensin II was shown to induce significantly CCN1 expression via its receptor type 1 (AT1R) [17, 18]. AT1R is expressed in all renal tubular and vascular segments and in mesan- gial cells $[19,20]$. It regulates tubular reabsorption and blood pressure, and seems to contribute to the development of renal dysfunction in cardiorenal syndrome [19, 21]. It remains unknown whether AT1R mediates renal expression of CCN1and if there are alterations of renal CCN1 expression in chronic renal dysfunction related to chronic circulatory failure, which corresponds to the cardiorenal syndrome type 2. The aim of this study was to evaluate CCN1 protein expression and localization in mouse kidney in basal conditions and in the model of chronic heart failure and to check whether IL-6 deficiency and AT1 receptor blockade affects renal CCN1 expression.

\section{Material and methods}

Animals. Male 14-16 week-old IL-6 gene knockout C57BL/ /6J IL-6-/-TMKopf (IL-6 KO) and wild-type (WT) reference strain $\mathrm{C} 57 \mathrm{BL} / 6 \mathrm{~J}$ mice were obtained from the Center for Experimental Medicine of the Medical University of Bialystok. Both strains were originally purchased from the Jackson Laboratories (West Grove, PA, USA). Animals were kept at constant temperature of $22^{\circ} \mathrm{C} \pm 1{ }^{\circ} \mathrm{C}$ in $12: 12$ darklight cycle with constant access to chow and water.

Myocardial infarction. Sixty four animals were subjected to coronary artery ligation to produce myocardial infarction. Twenty sex- and age-matched animals from both used strains subjected to sham operation were used as controls. The operation was performed as described previously with some modifications [22]. Briefly, animals were anaesthetized with isoflurane. After intubation of the trachea mice were put on a heated operating pad and ventilated with oxygenenriched air using rodent respirator (Minivent, Harvard Apparatus, USA). The chest was opened by left-sided incision in the fifth intercostal space, followed by opening of the pericardial sac and exposure of the anterior wall of the heart. In sham operated animals after 10 further minutes the chest was closed in layers with reposition of muscles. In order to induce myocardial ischemia in the remaining animals, the left coronary artery was ligated just proximally to its main bifurcation using Prolene 7.0 suture with atraumatic needle. Efficacy of myocardial ischemia was assessed visually as pale discoloration of the cardiac anterior wall. After closing of the chest wall, animals were further ventilated with oxygen-rich air without anaesthetic until spontaneous breath was evident (usually about 2 minutes). Mice were transferred to cages kept at $27^{\circ} \mathrm{C}$ and left for one hour to recover. Nineteen WT animals subjected to coronary artery ligation were for the next twelve weeks treated with telmisartan (\#T8949, Sigma, St. Louis, MO, USA) at a dose $1 \mathrm{mg} / \mathrm{kg} /$ day in drinking water [17] and constituted the MI-Telmisartan group, while the remaining mice after myocardial infarction (MI group) or sham operated (Sham) were not subjected to fur- 
ther treatment. All mice were sacrificed by cervical dislocation. Their hearts were excised and taken for further examinations, then the right kidney was fixed in isotonic PBS-buffered formalin and the left kidney was snap frozen in liquid nitrogen and stored at $-80^{\circ} \mathrm{C}$ until further analyses.

Myocardial dysfunction was diagnosed based on echocardiography performed eight weeks after surgery with the use of the M12L (14 mHz) ultrasound transducer. In addition gene expression of atrial natriuretic peptide (ANP) in the samples of the left ventricle myocardium was established using the twostep real time PCR method with the TaqMan Gene Expression Assay (Applied Biosystems, USA). Mice that did not show a visible area of akinesis in echocardiography and decrease of left ventricle ejection fraction below $35 \%$, or that did not present at least 2-fold increase of ANP gene expression in comparison to control group were excluded from the analyses. Forty three animals from three infarction groups (29 WT mice, of which 11 were treated with telmisartan and 14 IL-KO mice) and twenty sham-operated (10 WT and $10 \mathrm{IL}-6 \mathrm{KO}$ ) mice were included in the final analysis.

Genotyping. Genomic DNA for IL-6 genotyping was isolated from mouse tails using "Genomic mini" kit (A\&A Biotechnology, Poland) according to the enclosed protocol. PCR was performed using PCR Master Mix (2x) (Fermentas, Lithuania) and custom made primers (F 5'-CCATCCAGTTGCCTTCTTG-3', R 5'-AAGTGCATCATCGTTGTTCATAC-3'). After initial denaturation at $94^{\circ} \mathrm{C}$ over 5 minutes, amplification was performed in thirty PCR cycles under the following conditions: $94^{\circ} \mathrm{C} 20$ seconds, $52^{\circ} \mathrm{C} 30$ seconds, $72^{\circ} \mathrm{C} 3$ min., with final extension at $72^{\circ} \mathrm{C}$ for 10 minutes. Then, DNA was separated by electrophoresis on $1 \%$ agarose gel with ethidium bromide. GeneRuler DNA Ladder Mix (Fermentas, Lithuania) was used for PCR product size identification.

Western blotting. Western blotting procedure was performed as described previously [23]. Protein extracts were subjected to SDS-PAGE and blotted onto nitrocellulose membranes (BioRad, USA). Equal loading was confirmed using Ponceau Red staining (Sigma, USA). Primary antibodies recognizing mouse CCN1 were bought from Santa Cruz Biotechnology (\#sc-13100, Santa Cruz Biotechnology, Santa Cruz, CA, USA). Secondary antibodies were conjugated with horseradish peroxidase (Star54, Serotec, Oxford, UK). Blots were visualized using enhanced chemiluminescence reaction (Pierce, Rockford, IL, USA), and exposed on x-ray film (X-Omat Blue, Kodak, Rochester, NY). Results were scanned and quantified using Scion Image software (Scion Corporation, Frederick, MD, USA). The results of particular experiments were related to the expression of proteins in the control group, which was set as $100 \%$.

Immunohistochemical evaluation of CCN1 protein localization. Formalin-fixed, paraffin-embedded tissue blocks

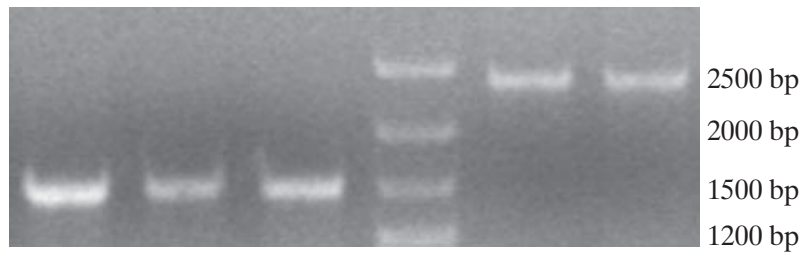

Figure 1. IL-6 genotyping of wild-type and IL- 6 deficient animals. DNA electrophoresis on agarose gel. Lanes from left: 1 to 3 - wild-type (C57BL/6J) animals; 4 - DNA ladder; 5 and $6-\mathrm{C} 57 \mathrm{BL} / 6 \mathrm{~J}^{\mathrm{IL}-6-/ / \mathrm{Kopf}}$ mice

were sectioned into $5 \mu \mathrm{m}$ slices. After deparafinization and rehydration, sections were pretreated with proteinase $\mathrm{K}$ solution and then blocked with $10 \%$ donkey serum in phosphate buffered saline (PBS) for 1 hour at room temperature. The endogenous peroxidase activity was blocked with $3 \% \mathrm{H}_{2} \mathrm{O}_{2}$. The primary antibodies against CCN1 (H-78, \#sc13100, SantaCruz Biotechnology, SantaCruz, CA, USA) were applied at 1:100 dilution in PBS for 90 minutes at room temperature. Then slides were incubated with proper secondary antibody (Star54, Serotec, Oxford, UK) conjugated with horseradish peroxidase at dilution 1:100 for one hour at room temperature. In the next step sections were rinsed with PBS and incubated with the DAB substrate (DAB Peroxidase Substrate Kit, Vector Laboratories, Burlingame, CA, USA) for 10 minutes. Nuclei were counterstained with hematoxylin. Negative control of staining was performed in the corresponding sections by omitting the primary antibody.

Ethical issues. The experimental procedures were carried out according to the European Council Directive of 24 November 1986 (6/609/EEC) and were approved by the Local Ethics Committee of Bialystok.

Statistics. Results were presented as mean \pm standard deviation (SD). U Mann-Whitney test was used for statistical analysis. A $P$ value lower than 0.05 was considered statistically significant.

\section{Results}

\section{IL-6 genotyping}

Material from wild-type animals yielded DNA fragments of the size ca. $1476 \mathrm{bp}$, whereas the respective DNA fragments from IL-6 KO animals included a fragment of neomycin cassette and were of the size ca. 2400 bp (Figure 1).

\section{Western blot analysis}

Western blotting analysis using an antibody against $\mathrm{CCN} 1$ revealed higher abundance of CCN1 protein 

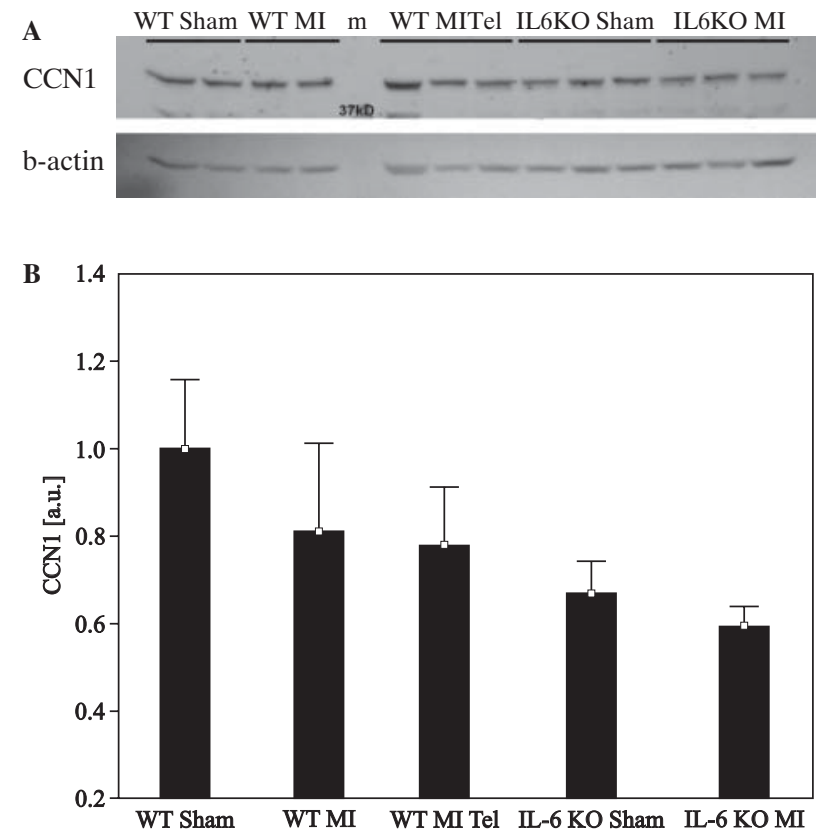

Figure 2. Relative abundance of CCN1 protein measured by Western blot. The measurements were performed on kidney samples obtained from sham-operated mice (Sham) and mice who underwent myocardial infarction 12 weeks earlier (MI), IL-6 deficient (IL-6 KO) or wild type (WT) mice, as well as from 'infarcted' mice treated with antagonist of AT1 receptor, telmisartan (Tel); (A) representative Western blot of CCN1 and beta-actin (reference);

(B) columns on the graph represent mean values \pm SEM obtained from 10-16 animals

in the kidney of WT sham-operated animals than in IL-6 deficient sham-operated mice (Figure 2), however this difference did not reach the level of statistical significance. Twelve weeks after myocardial infarction a slight decrease of CCN1 protein in the kidney of both WT and IL-6 KO mice was observed. Postoperative treatment of WT mice with an antagonist of AT1 receptor, telmisartan, did not change the level of CCN1 in kidney extracts.

\section{Immunohistochemical evaluation of CCN1}

In normal kidney from WT sham-operated mice $\mathrm{CCN} 1$ protein was expressed in both renal cortex and medulla. In the cortex, the strongest expression was observed in distal convoluted tubules and to a lesser degree in proximal convoluted tubules, especially in the brush border of tubular epithelium (Figure 3A). In the medulla, a similar intensity of CCN1 reaction as in proximal convoluted tubules was seen in the outer stripe of outer medulla, which corresponds to proximal straight tubules, and in the thick portion of
Henle's loop, as well as in the epithelium of collecting ducts. A strong CCN1-positive reaction was observed in smaller arteries (Figure 3B), however, not in large arteries. Moreover, CCN1-positive reaction was visible in some cells of glomeruli, probably podocytes ( Figure 3B).

Twelve weeks after myocardial infarction attenuation of CCN1-positive reaction throughout the renal cortex in both proximal and distal convoluted tubules, as well as in small arteries and glomeruli was observed (Figure 3C). In the outer stripe of outer medulla the intensity of reaction was not changed as compared to WT sham operated animals. Lower CCN1-positive staining in small arteries was noted in both sham operated and infracted IL-6 KO groups (Figure 3H).

In the group of animals subjected to myocardial infarction and treated with telmisartan, the characteristic distribution of $\mathrm{CCN} 1$ protein was observed in the kidney cortex. The intensity of CCN1-positive staining in straight proximal tubules was comparable to the WT infarcted group. CCN1 protein was almost absent in proximal convoluted tubules and in the inner medulla (Figure 3E and 3F), while strong CCN1positive staining was observed in distal convoluted tubules (Figure 3D). In the outer stripe of outer medulla the intensity of reaction was comparable to that of other groups of WT animals. Similarly to the WT infarcted group, less intensive CCN1-positive staining was observed in smaller arteries, with no reaction in large arteries, as compared with WT sham mice. Moreover, slight attenuation of CCN1-positive staining was observed in glomeruli.

In IL-6 deficient mice the intensity of CCN1-positive reaction and distribution of $\mathrm{CCN} 1$ protein in sham-operated animals (Figure 3G) and after myocardial infarction (Figure $3 \mathrm{H}$ ) did not differ. There were, however, some differences in CCN1 distribution as compared with WT animals: CCN1 was downregulated in proximal convoluted tubules and in tubules of the internal part of renal medulla. The intensity of CCN1-positive reaction in distal convoluted tubules was similar to WT animals. Also, differences in CCN1 staining between smaller and larger arteries were observed as described in the other groups of mice. Similarly to WT animals, a slight attenuation of CCN1 immunoreactivity in the glomeruli was observed in mice after infarction (Figure $3 \mathrm{G}$ and $3 \mathrm{H})$.

\section{Discussion}

Results of the present study indicate that $\mathrm{CCN} 1$ protein is distributed throughout mouse kidney and that 

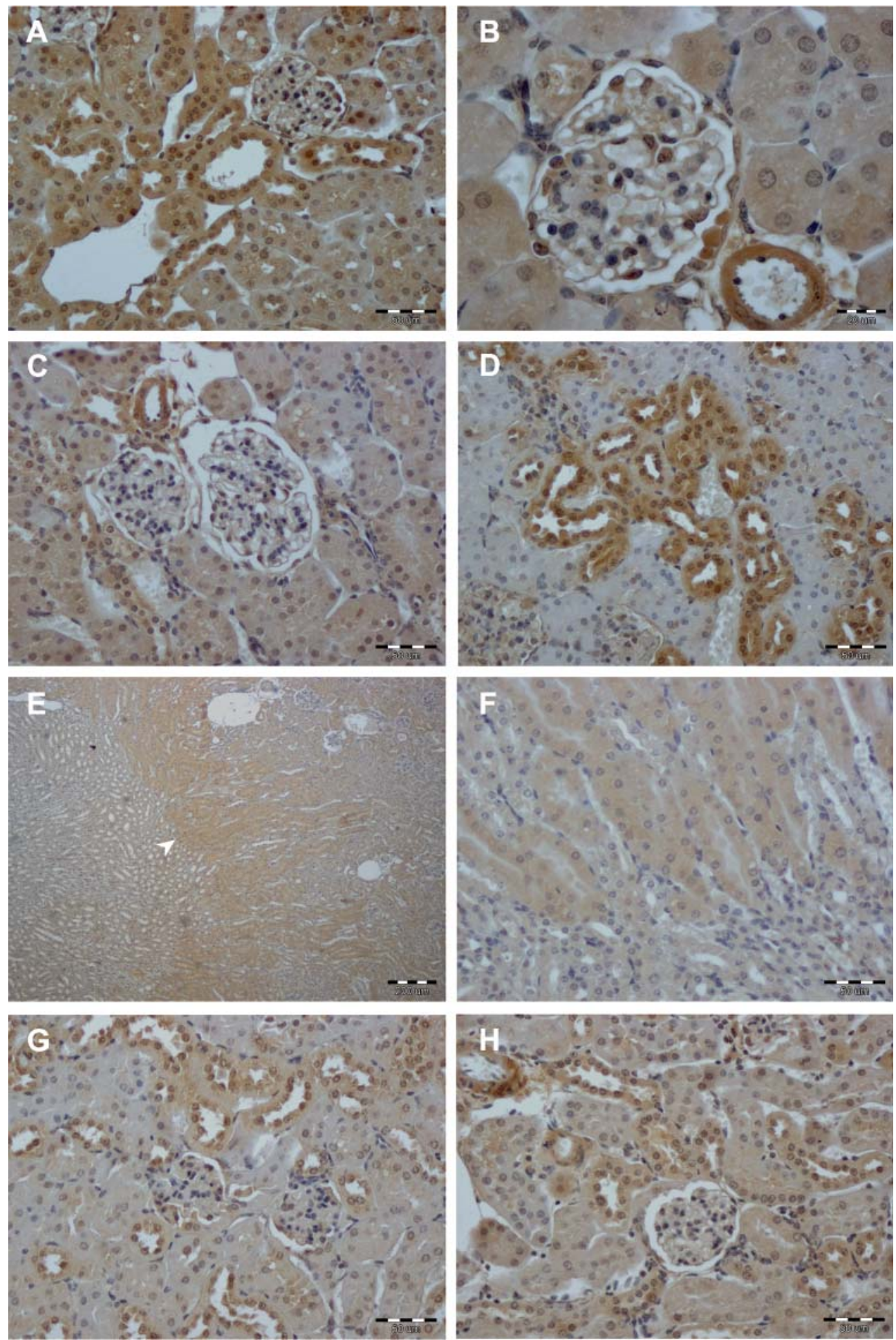

Figure 3. Immunohistochemical analyses of CCN1 protein expression in kidney from sham-operated and myocardial infarcted mice of IL-6 deficient (IL-6 KO) or wild type (WT) genotype. In sham-operated WT mice kidney the strongest reaction for CCN1 was seen in distal convoluted tubules $(\mathbf{A})$ and smaller arteries $(\mathbf{B})$, while weaker reaction was present in proximal convoluted tubules (A and B). CCN1 protein was also present in some podocytes (B). In the kidney from infarcted WT mice the reaction for CCN1 protein was weaker, however, its distribution was similar as in the shamoperated ones $(\mathbf{C})$. In kidney cortex from WT infarcted animals treated with telmisartan, a strong reaction for CCN1 protein was present in distal convoluted tubules, and there was no reaction in proximal convoluted tubules (D), while in the medulla positive CCN1 reaction was present in the outer stripe of outer medulla — arrowheads $(\mathbf{E}$ and $\mathbf{F}-$ higher magnification). Immunohistochemical reaction for CCN1 in IL-6 KO sham-operated (G) and IL-6 KO infarcted mice (H) was comparable and was weaker than in WT sham-operated and infarcted animals. Magnifications: A, C, D, F, G and H $-200 \times ; \mathrm{B}-400 \times, \mathrm{E}-40 \times$ 
the expression of CCN1 protein is lower in IL-6 deficient mice than in WT animals. The results of immunohistochemical evaluation of CCN1 protein are in accordance with those obtained by Western blotting. Although the differences in $\mathrm{CCN} 1$ protein quantity were small and statistically insignificant, differences in protein distribution between the investigated groups of mice were more pronounced. In all groups of animals the strongest immunohistochemical reaction for CCN1 was observed in distal convoluted tubules and in smaller arteries. The main difference in CCN1 distribution between the mice of the two examined genotypes was very week reaction in internal renal medulla and weaker reaction in proximal convoluted tubules in IL- 6 deficient mice in comparison to WT ones. Heart failure, evoked by myocardial infarction performed twelve weeks earlier, confirmed by the echocardiographic evaluation and by the expression of atrial natriuretic peptide in the myocardium of left ventricle, had only small effect on the expression of CCN1 protein in WT mice kidneys and had no effect in IL-6 deficient mice. Angiotensin II upregulates $\mathrm{CCN} 1$ synthesis in the cardiovascular system and this effect is blunted by blockade of the AT1 receptors [17, 18], however, up till now it was not known whether similar regulation occurs in the kidney. In pathological conditions AT1 receptor mediates upregulation of proinflammatory genes, including IL-6, adhesion molecules, monocyte chemoattractant protein-1 [24] and promotes accumulation of the extracellular matrix [25]. It was suggested that AT1 receptor mediates the AngII-induced activation of nuclear factor $\mathrm{kB}$ in the tubular epithelium [26]. In the experimental model of chronic heart failure syndrome in rats, activation of renal AT1R was shown to contribute to the development of progressive renal dysfunction [21].

In our study blocking of AT1 receptors with telmisartan did not have prominent influence on total CCN1 protein expression in infarcted WT mice, however, telmisartan treatment attenuated $\mathrm{CCN} 1$ expression in the proximal convoluted tubules, which may suggest a role of angiotensin II in the expression of CCN1 in this portion of the nephron.

There is only one study which demonstrated CCN1 expression in Balb/c mice normal kidney by the Western blotting method [14]. Unfortunately, authors of that study did not examine $\mathrm{CCN} 1$ protein distribution in mouse kidney, however, they evaluated the appearance of $\mathrm{CCN} 1$ protein both in mouse and rat kidney following 40 minutes of bilateral renal ischemia. $\mathrm{CCN} 1$ protein was detectable in the kidneys of normal and sham-operated Balb/c mice, and its levels increased at one hour after ischemia, peaked at four to eight hours, and CCN1 was still expressed at 24 hours after ischemia. Although the authors did not detect $\mathrm{CCN} 1$ protein expression in the kidney from rats subjected to sham surgery, they observed a dramatic upregulation of $\mathrm{CCN} 1$ protein following 40 minutes of bilateral renal ischemia. They also did not detect any CCN1 mRNA in the kidney of normal or sham-operated rats using in situ hybridization, however, two hours after ischemia they observed appearance of the signal in the outer stripe of the outer medulla and medullar rays, kidney regions which showed maximal tubular injury following renal ischemia/reperfusion. In that study CCN1 mRNA was primarily expressed in the cytoplasm of proximal straight tubules that showed mild injury, and was not detected in the inner medulla of normal or ischemic rat kidneys. Moreover, $\mathrm{CCN} 1$ protein was detected in urine three to six hours after renal ischemia, and its concentration peaked at six to nine hours, and decreased thereafter. Although the role of urinary CCN1 is unknown, the authors speculate that it might provide an important signal to a downstream nephron segment [6].

A second study, dealing with CCN1 expression in rat kidney, was performed in an experimental model of Thy-1 glomerulonephritis [15]. Contrary to the previous study, CCN1 protein expression, evaluated by in situ hybridization, was observed in normal rat kidney in the outer stripe of outer medulla, which corresponded to the proximal straight tubules. Moreover, intense expression of CCN1 protein was also observed in some afferent and efferent arterioles, however, not in larger vessels. At day 5 of experimental glomerulonephritis mRNA of CCN1 was detected on outer surfaces of glomerular basement membrane, which appeared to be podocytes. Similarly to the above studies performed on rats $[14,15]$, in all groups of mice evaluated in the present study we observed CCN1-positive staining in proximal straight tubules, and contrary to studies performed on rats, we found the strongest reaction in distal convoluted tubules. Moreover, contrary to rats, in mouse kidney we did not observe CCN1-positive staining in afferent and efferent arterioles, but in medium size arteries, while similarly as in rats we did not observe any reaction in large arteries. In addition, we detected CCN1-positive staining in mouse glomeruli, probably in podocytes, which supports earlier findings of Sawai et al. [15, 27] who detected CCN1 expression in cultured mouse podocytes.

Some differences in the pattern of CCN1 distribution in normal human kidney as compared to rodents were described by Sawai et al. [27]. CCN1 protein was expressed in podocytes, the thick ascending 
limb of Henle's loop, distal and proximal tubules, and collecting ducts in human kidney. Decreased CCN1 expression in human podocytes was observed in diseases affecting glomeruli, while the intensity of $\mathrm{CCN} 1$ staining in the tubular system was not altered [27]. CCN1 was reported to inhibit mesangial cell adhesion and migration and to promote podocyte differentiation and suppress podocyte migration in vitro [27]. Authors proposed that reduced CCN1 may affect glomerular structure and function by promoting mesangial migration leading to glomerulosclerosis and allowing for podocyte migration, that would cause glomerular crescent formation [27]. Biological effects of CCN1 depend on the specific membrane receptor and cell type. CCN1 acts on endothelial cells via integrin $\alpha \mathrm{v} \beta 3$ to promote adhesion [28]. CCN peptides are ligands of integrins $\alpha \mathrm{II} \beta 3$ in platelets and $\alpha \mathrm{v} \beta 3$ in monocytes [29, 30]. CCN1 also interacts with heparinsulfate-containing proteoglycans (HSPGs), including syndecan 4 and perlecan $[31,32]$. Integrins and HSPGs are essential for the adhesive and mitogenic function of $\mathrm{CCN}$ proteins [31, 32], and syndecan 4 and integrin $\alpha 6 \beta 1$ are necessary for CCN1-mediated induction of apoptosis in fibroblasts [32]. $\mathrm{CCN} 1$ is involved in the inflammatory response by activation of proinflammatory genetic program in murine macrophages throughout $\alpha_{\mathrm{M}} \beta_{2}$ and syndecan- 4 and is able to directly induce the expression of certain proinflammatory genes, such as TNF- $\alpha$ and IP-10 genes, whereas upregulation of other proinflammatory genes, including IL- $1 \beta 1$ and IL- 6 , requires the induction of TNF- $\alpha$ as a mediator [33]. Therefore multiple biological activities can be elicited through the interaction of discrete domains of the CCN proteins with different cell membrane receptors.

CCN1 protein expression represents an immediate early gene response, therefore, regulation of this protein in the kidney and other organs was mainly described in experimental models of acute diseases $[14,15,17]$. Acute kidney ischemia/reperfusion upregulates CCN1 expression [14], so it may be hypothesized that early after myocardial infarction there is also transient induction of CCN1, which might have delayed alterations of kidney structure and function. In our model of chronic heart failure we observed only small changes in the CCN1 expression in mouse kidney, with a tendency for downregulation of CCN1 twelve weeks after myocardial infarction. Unfortunately, in the present study we have not evaluated the possible dynamics of CCN1 expression in the kidney, and there were no evident structural changes in kid- neys under light microscopy to support the abovementioned hypothesis.

The present study has demonstrated for the first time the expression and localization of $\mathrm{CCN} 1$ protein both in wild type and IL-6 deficient C57BL/6J mouse kidney, both under basic conditions and in experimental chronic heart failure evoked by myocardial infarction performed twelve weeks earlier. We have shown that the distribution of CCN1 protein in mouse kidney presents a pattern different from that seen in rats, bearing more resemblance to the one seen in humans. In contrast to rats, in all groups of mice of both genotypes, the strongest immunohistochemical CCN1-positive reaction was shown in distal convoluted tubules, and in smaller arteries, while similarly to rats, CCN1 protein was also present in proximal straight tubules $[14,15,27]$. In IL-6 deficient mice CCN1 expression, estimated by Western blot technique, was in the kidney insignificantly lower in comparison to WT animals. Experimental heart failure in mouse kidney caused only slight alterations of CCN1 protein expression. These alterations affected mainly proximal convoluted tubules and the inner part of kidney medulla.

Diminished CCN1 expression may be of importance in view of the increased fibrosis of the renal interstitium after myocardial infarction [3]. CCN1 controls fibrosis by inducing senescence of myofibroblasts [13], and decreased CCN1 expression may promote renal fibrosis after infarction. Certainly, CCN1 is not the only one nor the most important regulator of fibrosis since the most important one seems to be AngII, as its blockade prevents development of renal fibrosis [25]. Our results suggest that AngII may considerably downregulate $\mathrm{CCN} 1$ in the kidney proximal tubules.

In summary, we have shown that the expression of CCN1 protein in IL-6 deficient mouse kidney was slightly lower in comparison to WT animals, and that heart failure following myocardial infarction induced only small alterations of $\mathrm{CCN} 1$ protein expression in mouse kidney.

\section{Acknowledgements}

This study was supported by grants N401 103 31/2289 of the Polish Ministry of Science and Higher Education and funds from the National Science Center DEC-2011/01/B/NZ4/04862. Karol Kamiński was supported by grant from the Foundation for Polish Science $05 / 11$. 


\section{References}

1. Ronco C, Haapio M, House AA, Anavekar N, Bellomo R. Cardiorenal syndrome. J Am Coll Cardiol. 2008;52:1527-1539.

2. Hillege HL, van Gilst WH, van Veldhuisen DJ et al. Accelerated decline and prognostic impact of renal function after myocardial infarction and the benefits of ACE inhibition: the CATS randomized trial. Eur Heart J. 2003;24:412-420.

3. Lekawanvijit S, Kompa AR, Zhang Y et al. Myocardial infarction impairs renal function, induces renal interstitial fibrosis, and increases renal KIM-1 expression: implications for cardiorenal syndrome. Am J Physiol Heart Circ Physiol. 2012;302:H1884-1893.

4. Kaminski KA, Kozuch M, Bonda T et al. Coronary sinus concentrations of interleukin 6 and its soluble receptors are affected by reperfusion and may portend complications in patients with myocardial infarction. Atherosclerosis. 2009;206: 581-587.

5. Hsiao PG, Hsieh CA, Yeh CF et al. Early prediction of acute kidney injury in patients with acute myocardial injury. J Crit Care. 2012;27:525 e521-527.

6. Torre-Amione G, Kapadia S, Benedict C et al. Proinflammatory cytokine levels in patients with depressed left ventricular ejection fraction: a report from the Studies of Left Ventricular Dysfunction (SOLVD). J Am Coll Cardiol. 1996;27:1201-1206.

7. Bozkurt B, Mann DL, Deswal A. Biomarkers of inflammation in heart failure. Heart Fail Rev. 2010;15:331-341.

8. Pecoits-Filho R, Lindholm B, Axelsson J, Stenvinkel P. Update on interleukin-6 and its role in chronic renal failure. Nephrol Dial Transplant. 2003;18:1042-1045.

9. Naito K, Anzai T, Yoshikawa T et al. Impact of chronic kidney disease on postinfarction inflammation, oxidative stress, and left ventricular remodeling. J Card Fail. 2008;14:831-838.

10. Kishimoto T, Akira S, Narazaki M, Taga T. Interleukin-6 family of cytokines and gp130. Blood. 1995;86:1243-1254.

11. Klein R, Stiller S, Gashaw I. Epidermal growth factor upregulates endometrial CYR61 expression via activation of the JAK2/STAT3 pathway. Reprod Fertil Dev. 2012;24:482-489.

12. Perbal B. CCN proteins: multifunctional signalling regulators. Lancet. 2004;363:62-64.

13. Lau LF. CCN1/CYR61: the very model of a modern matricellular protein. Cell Mol Life Sci. 2011;68:3149-3163.

14. Muramatsu Y, Tsujie M, Kohda Y et al. Early detection of cysteine rich protein 61 (CYR61, CCN1) in urine following renal ischemic reperfusion injury. Kidney Int. 2002;62:1601-1610.

15. Sawai K, Mori K, Mukoyama M et al. Angiogenic protein Cyr61 is expressed by podocytes in anti-Thy- 1 glomerulonephritis. J Am Soc Nephrol. 2003;14:1154-1163.

16. Chen Y,Du XY. Functional properties and intracellular signaling of CCN1/Cyr61. J Cell Biochem. 2007;100:1337-1345 .

17. Hilfiker-Kleiner D, Kaminski K, Kaminska A et al. Regulation of proangiogenic factor $\mathrm{CCN} 1$ in cardiac muscle: impact of ischemia, pressure overload, and neurohumoral activation. Circulation. 2004;109:2227-2233.
18. Hilfiker A, Hilfiker-Kleiner D, Fuchs M et al. Expression of CYR61, an angiogenic immediate early gene, in arteriosclerosis and its regulation by angiotensin II. Circulation. 2002;106:254-260.

19. Bouby N, Hus-Citharel A, Marchetti J et al. Expression of type 1 angiotensin II receptor subtypes and angiotensin II-induced calcium mobilization along the rat nephron. $\mathrm{J} \mathrm{Am}$ Soc Nephrol. 1997;8:1658-1667.

20. Chansel D, Czekalski S, Pham P, Ardaillou R. Characterization of angiotensin II receptor subtypes in human glomeruli and mesangial cells. Am J Physiol. 1992;262:F432-441.

21. Homma T, Sonoda H, Manabe K et al. Activation of renal angiotensin type 1 receptor contributes to the pathogenesis of progressive renal injury in a rat model of chronic cardiorenal syndrome. Am J Physiol Renal Physiol. 2012;302:F750-761.

22. Lutgens E, Daemen MJ, de Muinck ED et al. Chronic myocardial infarction in the mouse: cardiac structural and functional changes. Cardiovasc Res. 1999;41:586-593.

23. Kaminski KA, Kozuch M, Bonda TA et al. Effect of interleukin 6 deficiency on the expression of Bcl-2 and Bax in the murine heart. Pharmacol Rep. 2009;61:504-513.

24. Ruiz-Ortega M, Lorenzo O, Suzuki Y, Ruperez M,Egido J. Proinflammatory actions of angiotensins. Curr Opin Nephrol Hypertens. 2001;10:321-329.

25. Ruiz-Ortega M, Ruperez M, Esteban V et al. Angiotensin II: a key factor in the inflammatory and fibrotic response in kidney diseases. Nephrol Dial Transplant. 2006;21:16-20.

26. Ruiz-Ortega M, Lorenzo O, Ruperez M, Blanco J,Egido J. Systemic infusion of angiotensin II into normal rats activates nuclear factor-kappaB and AP-1 in the kidney: role of AT(1) and AT(2) receptors. Am J Pathol. 2001;158:1743-1756.

27. Sawai K, Mukoyama M, Mori K et al. Expression of CCN1 (CYR61) in developing, normal, and diseased human kidney. Am J Physiol Renal Physiol. 2007;293:F1363-1372.

28. Kireeva ML, Lam SC, Lau LF. Adhesion of human umbilical vein endothelial cells to the immediate-early gene product Cyr61 is mediated through integrin alphavbeta3. J Biol Chem. 1998;273:3090-3096.

29. Jedsadayanmata A, Chen CC, Kireeva ML, Lau LF, Lam SC. Activation-dependent adhesion of human platelets to Cyr61 and Fisp12/mouse connective tissue growth factor is mediated through integrin alpha(IIb)beta(3). J Biol Chem. 1999;274:24321-24327.

30. Schober JM, Lau LF, Ugarova TP, Lam SC. Identification of a novel integrin alphaMbeta2 binding site in CCN1 (CYR61), a matricellular protein expressed in healing wounds and atherosclerotic lesions. J Biol Chem. 2003;278:25808-25815.

31. Chen Y, Abraham DJ, Shi-Wen X et al. CCN2 (connective tissue growth factor) promotes fibroblast adhesion to fibronectin. Mol Biol Cell. 2004;15:5635-5646.

32. Todorovicc V, Chen CC, Hay N, Lau LF. The matrix protein CCN1 (CYR61) induces apoptosis in fibroblasts. J Cell Biol. 2005; 171:559-568.

33. Bai T, Chen CC, Lau LF. Matricellular protein CCN1 activates a proinflammatory genetic program in murine macrophages. J Immunol. 2010;184:3223-3232.

Submitted: 20 August, 2012 Accepted after reviews:12 March, 2013 